\title{
Methodology for retinal photography and assessment of diabetic retinopathy: the EURODIAB IDDM Complications Study
}

\author{
S.J.Aldington ${ }^{1}$, E.M.Kohner ${ }^{1}$, S.Meuer ${ }^{2}$, R. Klein ${ }^{2}$, A.K. Sjølie ${ }^{3}$ for the EURODIAB IDDM Complications Study \\ Group \\ ${ }^{1}$ Diabetic Retinopathy Unit, Department of Medicine, Royal Postgraduate Medical School, London, UK \\ ${ }^{2}$ Department of Ophthalmology, University of Wisconsin, Wisconsin, USA \\ ${ }^{3}$ Department of Ophthalmology, Århus University Hospital, Århus, Denmark
}

Summary We present the methodology for $45^{\circ}$ retinal photography and detail the development, application and validation of a new system of $45^{\circ}$ field grading standards for the assessment of diabetic retinopathy. The systems were developed for the EURODIAB IDDM Complications Study, part of a European Community funded Concerted Action Programme into the epidemiology and prevention of diabetes (EURODIAB). Assessment of diabetic retinopathy was carried out centrally by a trained reader of colour retinal photographs using the newly-developed system. The system proved to be acceptably accurate, repeatable and relatively simple to apply. It compared well with the recognised 'gold standard' 7field $30^{\circ}$ stereo photography (assessed using a modified Airlie House classification scheme), against which the new system was validated in a series of 48 eyes. Selection was as a stratified random sample based on clinical retinopathy status: 5 , no retinopathy; 25 , non-proliferative retinopathy; 16 , proliferative or photocoagulated; plus 2, eyes with potentially confounding lesions (vein occlusion). Simple presence of retinal lesions was correctly detected by both systems in 43 of the 48 eyes, giving $100 \%$ agreement on detection. Both systems correctly identified the two known cases of confounding vein occlusion. In eyes with diabetic retinopathy $(n=41)$, when severity was expressed in three groups: mild background, moderate/severe background and proliferative/ photocoagulated, at least one grader (out of five) using the new system matched the verified results in 38 out of $41(93 \%)$ eyes and three or more graders matched in $31(76 \%)$ eyes. Individually the five graders' 2 -field allocations agreed well with the verified levels (median number of agreements 37, range 2843). Repeatability was assessed by measures of within and between observer variation using randomly selected samples of $10 \% \quad(n=252$ eyes $)$ and $5 \%$ ( $n=123$ eyes) of the main study, respectively, expressed as a resultant kappa value for chance-corrected proportional agreement. Within observer assessment yielded a kappa of 0.85 and between observers a value of 0.83 ; indicating very good agreement for both measures. The method is particularly useful for large epidemiological studies, in which participating centres have a limited experience in retinal photography. [Diabetologia (1995) 38: 437-444]

Key words Insulin-dependent diabetes, diabetic retinopathy, retinal photography, grading scheme.
Received: 27 April 1994 and in revised form: 6 September 1994

Corresponding author: Mr. S. J. Aldington, Diabetic Retinopathy Unit, Royal Postgraduate Medical School, Du Cane Road, London W12 ONN, UK

Abbreviations: HMA, Haemorrhages and microaneurysms; ETDRS, Early Treatment Diabetic Retinopathy Study.
A major component of the EURODIAB Concerted Action programme has been the study of the frequency of complications of insulin-dependent diabetes mellitus in Europe. The overall aims and primary results of this study have been described previously [1].

In this report we present the methodology for $45^{\circ}$ retinal photography and detail the development of a novel assessment system for diabetic retinopathy from $45^{\circ}$ photographs. 

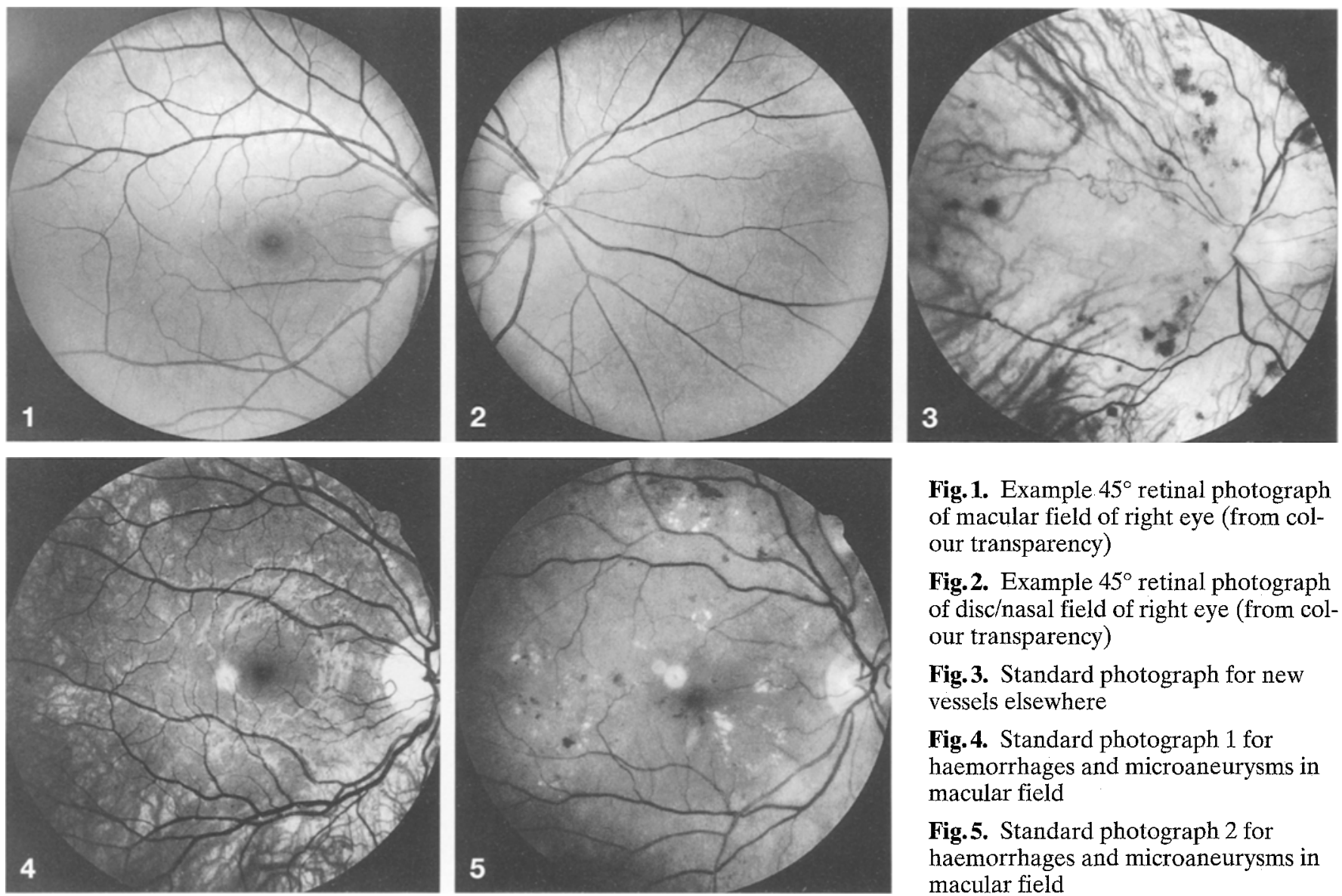

Fig. 1. Example $45^{\circ}$ retinal photograph of macular field of right eye (from colour transparency)

Fig. 2. Example $45^{\circ}$ retinal photograph of disc/nasal field of right eye (from colour transparency)

Fig. 3. Standard photograph for new vessels elsewhere

Fig. 4. Standard photograph 1 for haemorrhages and microaneurysms in macular field

Fig. 5. Standard photograph 2 for haemorrhages and microaneurysms in macular field

\section{Subjects, materials and methods}

Thirty-one hospital-based European clinical diabetes centres participated in the EURODIAB IDDM Complications Study. Two centres did not have access to suitable retinal photography equipment and were unable to participate in this aspect.

A relatively simple photographic protocol was devised such that the centres could produce material suitable for the assessment of diabetic retinopathy from retinal photographs, taken with wide-angle cameras.

\section{Photographic methods}

Prior to retinal photography, the patient's pupils were dilated using tropicamide $1 \%$ and additional epinephrine $10 \%$ if required, repeated if pupils did not reach at least $6 \mathrm{~mm}$ in diameter.

Colour retinal photographs, with a suitable $45^{\circ}$ retinal camera of the centre's choice, were taken by a trained operator of two fields of both eyes:

Macular field: positioned such that the exact centre of the optic disc lay at the nasal end of the horizontal meridian of the field of view.

Disc/nasal field: such that the optic disc was positioned one disc-diameter in from the temporal edge of the field, on the horizontal meridian.
Examples of the two retinal fields photographed (of the right eye) are shown as Figures 1 and 2, respectively.

The two fields recorded a retinal view (allowing for overlap) of approximately $80^{\circ}$ horizontally by $45^{\circ}$ vertically, therefore clinically significant and/or immediately sight-threatening lesions of diabetic retinopathy were detectable in most instances. In order to test this, comparison with the recognised 'gold standard' photographic grading system, 7-field $30^{\circ}$ stereo photography was carried out.

Photographic film was standardised on 24-exposure Kodachrome 64 ISO (Kodak, Rochester, N. Y., USA) transparency film (diapositive), purchased in bulk, stored and supplied centrally.

Retinal photographers received written instructions covering all aspects of the procedures, supplemented by practical training sessions. Additionally, they were provided with a 'quick reference guide' suitable for permanent positioning close to their fundus camera.

Photographers were required to take one picture of the patient's identification label, followed by one photograph each of the Macular then Disc/nasal field of right then left eyes. Four patients (approximately 20 exposures) could therefore be photographed on each 24-exposure film.

An optional sixth frame was allowed per patient. This gave the photographer the opportunity to repeat one retinal field if they considered that it would not be of sufficient quality for assessment. Alternatively the sixth frame could be used to document lesions (for instance peripheral neovascularisation), occurring only outside the standard photographic fields.

After film processing, the photographs for each patient were placed in a clear plastic pocketed storage sheet. The pa- 
tient's identification number and date of photography were written onto the storage sheet.

Completed storage sheets of retinal photographs were mailed at intervals to the Coordinating Centre at University College Hospital, London, UK, for forwarding in batches to the Diabetic Retinopathy Grading Centre at the Hammersmith Hospital, London, UK for assessment.

\section{Training methods}

A series of training meetings was organised during 1989-1990 at a number of European locations. Each centre was required to nominate relevant staff to attend at least one training meeting, where instruction in all aspects of the organisation, measurements and investigations was given.

The participants were instructed in the photographic protocol and each was required to produce acceptable retinal photographs of a volunteer subject during the training session.

After completion of the training but prior to commencement of the main clinical study, centres were required to submit a 'dry run' sample of photographs on four patients (not included in the main study sample) to demonstrate ability and understanding.

\section{Assessment methods}

Derivation of the system. It was necessary to develop a system of assessment for the EURODIAB. The chosen system was based on the principles of the Modified Airlie House classification scheme, as used for the Diabetic Retinopathy Study and subsequent derivatives [2-5]. Thereby, diabetic retinopathy lesions are assessed against one or more standard photographs for that lesion.

Subsequently, grades for each lesion type are used to produce a combinatorial, overall severity level for the eye, indicative of likelihood of progression [6].

To produce the standard photographs, a series of routine diabetic retinopathy clinic patients were photographed at the Hammersmith Hospital. From these, individual $45^{\circ}$ pictures were chosen, each demonstrating one or more particular lesions of diabetic retinopathy in a range of severity to follow the natural history of the disease process.

It proved more practical in some situations to utilise the concept of a standard measure rather than a standard photograph for certain lesions. Pre-retinal haemorrhage, vitreous haemorrhage and scars of photocoagulation utilise the qualitative measure of " $50 \%$ of field involved", as a standard measure, rather than a standard photograph. This procedure was also consistent with similar aspects of the Early Treatment Diabetic Retinopathy Study (ETDRS) protocol [7].

Lesions assessed. The lesions assessed in the EURODIAB system are shown in Table 1, with their corresponding grading standards. For some lesions, a single standard photograph was chosen. This gave possible gradings for that lesion as follows: 0 , lesion absent; 1 , lesion questionably present; 2 , definitely present but $<$ standard photograph; 3 , lesion present $\geq$ standard photograph.

As an example, the standard photograph for new vessels elsewhere is shown in Figure 3.

Lesions for which two standard photographs were chosen were allocated to an extended scale as follows: 0 , lesion absent; 1 , lesion questionably present; 2 , definitely present but $<$ standard photograph 1; 3, lesion present $\geq$ standard
Table 1. Lesions of diabetic retinopathy assessed

\begin{tabular}{|c|c|c|c|}
\hline Lesion & $\begin{array}{l}\text { Abbre- } \\
\text { viation }\end{array}$ & Field(s) & Standard(s) \\
\hline $\begin{array}{l}\text { Haemorrhages and } \\
\text { microaneurysms }\end{array}$ & HMA & $\begin{array}{l}\mathrm{M} \\
\mathrm{D} / \mathrm{N}\end{array}$ & $\begin{array}{l}\mathrm{SP} 1+\mathrm{SP} 2 \\
\mathrm{SP} 1+\mathrm{SP} 2\end{array}$ \\
\hline Hard exudates & $\mathrm{HE}$ & $\begin{array}{l}M \\
D / N\end{array}$ & $\begin{array}{l}\mathrm{SP} 1+\mathrm{SP} 2 \\
\mathrm{SP} 1+\mathrm{SP} 2\end{array}$ \\
\hline Cotton wool spots & CWS & $\begin{array}{l}\mathrm{M} \\
\mathrm{D} / \mathrm{N}\end{array}$ & $\begin{array}{l}\mathrm{SP} 1+\mathrm{SP} 2 \\
\mathrm{SP} 1+\mathrm{SP} 2\end{array}$ \\
\hline $\begin{array}{l}\text { Intraretinal microvas- } \\
\text { cular abnormalities }\end{array}$ & IRMA & $\begin{array}{l}M \\
D / N\end{array}$ & $\begin{array}{l}\mathrm{SP} 1+\mathrm{SP} 2 \\
\mathrm{SP} 1+\mathrm{SP} 2\end{array}$ \\
\hline Venous beading & VB & $\mathrm{M}+\mathrm{D} / \mathrm{N}$ & SP1 \\
\hline New vessels elsewhere & NVE & $\mathrm{M}+\mathrm{D} / \mathrm{N}$ & SP1 \\
\hline $\begin{array}{l}\text { Fibrous proliferation } \\
\text { elsewhere }\end{array}$ & FPE & $\mathrm{M}+\mathrm{D} / \mathrm{N}$ & $\mathrm{SP} 1+\mathrm{SP} 2$ \\
\hline New vessels disc & NVD & $\mathrm{D} / \mathrm{N}$ & SP1 \\
\hline Fibrous proliferation disc & FPD & $\mathrm{D} / \mathrm{N}$ & $\mathrm{SP} 1+\mathrm{SP} 2$ \\
\hline Pre-retinal haemorrhage & PRH & $\mathrm{M}+\mathrm{D} / \mathrm{N}$ & SM1 \\
\hline Vitreous haemorrhage & VH & $\mathrm{M}+\mathrm{D} / \mathrm{N}$ & SM1 \\
\hline Scars of photocoagulation & PC & $\mathrm{M}+\mathrm{D} / \mathrm{N}$ & SM1 \\
\hline
\end{tabular}

M, Macular field; D/N, Disc/nasal field; SP, standard photograph; SM, standard measure

photograph 1 but $<$ standard photograph $2 ; 4$, lesion present $\geq$ standard photograph 2.

Standard photograph 1 and 2 for haemorrhages and microaneurysms (HMA) in the Macular field are shown in Figures 4 and 5 , respectively.

The grading system utilised the ETDRS concept of grade 1 for 'questionable presence' of a lesion. This was defined as:

"the presence of a retinal lesion, the identity of which the grader was $>50 \%$ but $<90 \%$ sure was the lesion type currently being assessed" [7].

Assessment of four lesions. HMA, hard exudates, cotton wool spots and intraretinal microvascular abnormalities was against standards appropriate for the field in which it was being assessed. Other lesions were assessed against standards common to both fields. Lesions specific to the optic disc (new vessels of the disc and fibrous proliferations of the disc) were only assessed in the Disc/nasal field; as the only field in which the whole of the optic disc was visible.

The 23 chosen standard photographs were placed into three A5 storage sheets, identical to those used for clinical photographs. Sheet 1 of standards related to lesions assessed specifically in the Macular field, sheet 2 specifically for the Disc/nasal field and sheet 3 those standards common to both fields.

The use of identical storage media for clinical photographs and standards allowed immediate, direct comparisons to be made between the two under the same conditions.

Techniques of assessment. Viewing of both clinical and standard photographs was carried out utilising a self-contained retroprojection viewer, marketed for such purposes. The Slidex H-1 Projector (Slidex Corp., Tokyo, Japan) used for assessment projects clear, bright images from 35 -mm format transparencies onto the self-contained high quality back projection screen. Linear magnification was $11 \times$ to the screen with the entire $45^{\circ}$ field of the image visible.

Positioning of the particular image was by virtue of the adjustable glass 'stage' on which the storage sheets (and hence 
images) lay for projection. The stage was large enough to accommodate one entire set of patient photographs plus any one of the three sheets of grading standards.

A single, trained diabetic retinopathy assessor from the Hammersmith Hospital Grading Centre graded all the material over a 14-month period. The grader had no access to any information regarding the patient - specifically the gender, age and diabetes duration.

Photographs were identified by the patient's unique identification number and the date of photography. Assessment was recorded onto a grading form designed specifically for this study.

Quality assessment. On receipt, photographic quality and adherence to protocol were assessed. Focus and clarity of each field were assessed relative to ETDRS standard photograph 14 [7]. Any field not meeting this quality level was judged unassessable and it was not graded further. Quality assessments were annotated on the grading form as good, fair or poor (not assessable).

Retinopathy assessment. On each assessable field, lesions of diabetic retinopathy listed in Table 1 were graded. As a consequence of not taking stereo retinal photographs, it was not possible to assess retinal thickening and elevation directly, therefore maculopathy per se was not gradable in this study.

Grading results were recorded as numerical values into the relevant box on the grading form. Allowable grades for each lesion type in each field were pre-printed on the grading forms to assist the reader. All boxes on the grading form were filled, including zero values. Presence of any visible non-diabetic ocular conditions was also noted.

Both eyes were assessed consecutively, with the right eye always being graded first. Similarly, the Macular field of an eye was graded before the corresponding Disc/nasal field.

Completed grading forms were sent to the Coordinating Centre with copies retained in the Grading Centre.

Retinopathy level calculation. The detailed grading of retinopathy lesions was used to calculate an overall retinopathy level per eye. The concept is that groups of lesions, when occurring together, are broad indicators of progression of diabetic retinopathy towards proliferation and high-risk characteristics [6]. The higher the level assigned to an eye, the greater the risk. Such levels assigned in this study are shown in Table 2 . Implicit in such allocation systems is that the eye does not have any features of retinopathy of a higher level. In effect therefore the allocation is carried out "top down" i.e. not level 5 (proliferative), not level 4 (photocoagulated), not level 3 (pre-proliferative), but level 2 (moderate background) etc.

The allocation of severity level to assessed eyes was carried out automatically by a computer program, subsequent to data from the detailed gradings being entered onto computer in the Coordinating Centre. The principal advantage in using this type of system is that the algorithms assigning characteristics to the various levels can subsequently be changed, without affecting the original data on the grading of lesions.

In general, the levels expressed in Table 2 were consistent with clinical severity of diabetic retinopathy. Level 0 denoted absence of diabetic retinopathy. Level 1 was used to denote those eyes with minimal (background) retinopathy, with or without the presence of hard exudates. Level 2 characterised those eyes with more moderate to severe retinal haemorrhage/microaneurysms (HMA) in either of the two fields, or HMA in the presence of early lesions indicative of ischaemia (cotton wool spots, intraretinal microvascular abnormalities and/or venous beading). Level 3 was assigned to eyes display-
Table 2. Allocated retinopathy levels and causative features

\begin{tabular}{|c|c|c|}
\hline Level & & Retinopathy Features \\
\hline Level 0 & & No retinopathy \\
\hline Level 1 & & $\begin{array}{l}\text { Minimal non-proliferative retinopathy: } \\
\text { HMA = Grade } 2-3 \text { in } 1 \text { or } 2 \text { fields and/or } \\
\mathrm{HE}=\text { Grade } 2-4 \text { in } 1 \text { or } 2 \text { fields }\end{array}$ \\
\hline Level 2 & OR & $\begin{array}{l}\text { Moderate non-proliferative retinopathy: } \\
\text { HMA = Grade } 4 \text { in only } 1 \text { field } \\
\text { HMA = Grade } 2-3 \text { in } 1 \text { or } 2 \text { fields plus: } \\
\text { CWS = Grade } 2-3 \text { in } 1 \text { or } 2 \text { fields and/or } \\
\text { IRMA = Grade } 2 \text { in } 1 \text { or } 2 \text { fields and/or } \\
\text { VB = Grade } 2 \text { in } 1 \text { or } 2 \text { fields }\end{array}$ \\
\hline Level 3 & OR & $\begin{array}{l}\text { Severe non-proliferative (pre-proliferative) } \\
\text { HMA = Grade } 4 \text { in both fields } \\
\text { HMA = Grade } 2-4 \text { in } 1 \text { or } 2 \text { fields plus: } \\
\text { CWS = Grade } 4 \text { in } 1 \text { or } 2 \text { fields and/or } \\
\text { IRMA = Grade } 3 \text { in } 1 \text { or } 2 \text { fields and/or } \\
\text { VB = Grade } 3 \text { in } 1 \text { or } 2 \text { fields }\end{array}$ \\
\hline Level 4 & & $\begin{array}{l}\text { Photocoagulated: } \\
\text { Scars of photocoagulation in any field. }\end{array}$ \\
\hline Level 5 & & $\begin{array}{l}\text { Proliferative: } \\
\text { Any of: } \\
\text { New vessels (disc or elsewhere) } \\
\text { Fibrous proliferations (disc or elsewhere) } \\
\text { Pre-retinal haemorrhage } \\
\text { Vitreous haemorrhage }\end{array}$ \\
\hline
\end{tabular}

For abbreviations, see Table 1

ing classic pre-proliferative lesions with more advanced ischaemic features or severe HMA throughout the eye. Retinal scars due to previous photocoagulation treatment caused an eye to be allocated to Level 4 . The most severe group, Level 5, was used to denote eyes having features of proliferative diabetic retinopathy; new vessels, fibrous proliferations and pre-retinal or vitreous haemorrhage.

\section{Data handling}

To facilitate the safe handling of patient material, progress of photosets through the grading system was handled via a computerised database (Smart Integrated System, Innovative Software Inc., Lenexa, KS, USA). Retinopathy data from completed grading forms were computerised at the Coordinating Centre via a proprietary statistical recording package, SAS.

Copies of completed grading forms were provided for the relevant clinical centre as a report on the retinopathy findings on their patient.

\section{Statistical analysis}

Results are presented as number (\%), median (95\% CI) or mean (SD) as appropriate. Agreement is expressed by weighted or unweighted kappa value. 


\section{Results}

To validate this newly-developed system, it was prudent to compare it with one pre-existing and widely accepted and also to carry out an amount of regrading for quality assurance.

\section{Validation of standards}

A series of 24 diabetic clinic patients attending Århus University Hospital, not included in the EURODIAB Study group, were photographed by a single, experienced retinal photographer. Subjects received both $45^{\circ}$-field then $30^{\circ}$ 7-field stereo photography at the same visit.

The $30^{\circ}$ stereo images, taken as per ETDRS Manual of Operations for colour 7-field stereoscopic retinal photography [2] were assessed by two independent experienced observers; one from the Hammersmith Grading Centre, the second from the Fundus Photograph Reading Centre, Madison, Wis., USA. Assessment of $30^{\circ}$ photographs was carried out using the protocol for the Wisconsin Epidemiologic Study of Diabetic Retinopathy (WESDR) [3].

The $45^{\circ}$ photographs were independently assessed by five graders using the protocol as described herein.

Subsequently, direct comparisons were carried out between the results of the five $45^{\circ}$ gradings and two $30^{\circ}$ gradings, with concurrent examination of both sets of photographs.

For the purposes of assessing the accuracy of the 2field $45^{\circ}$ gradings, the results from the 7 -field $30^{\circ}$ gradings were considered to be the 'gold standard' in all cases except in two eyes. In one of these, new vessels were detected nasally to the optic disc on the $45^{\circ}$ photographs but this field was not represented in the $30^{\circ}$ standard fields. The second case was one in which cotton wool spots were detected on the $45^{\circ}$ photographs, yet the corresponding $30^{\circ}$ field was judged unassessable (though re-examination of the photographs did show the lesions). The corrected 'verified' levels therefore has been subsituted for that routinely assigned by the $30^{\circ}$ gradings for these two eyes, all others remaining.

Validation of standards - results. Both eyes of 24 patients were photographed on both $45^{\circ}$ and $30^{\circ}$ systems. Five eyes (of three patients) were assessed to have no retinal lesions on both systems by all graders. The remaining 43 eyes of 22 patients were judged to have retinal lesions present by both grading systems, giving total crude concurrence on detection.

After completion of grading using the relevant system and the assignment of overall retinopathy levels per eye, the results were contracted using the algorithms shown in Table 3, to allow comparison between the dissimilar values from the two systems.
Tahle 3. Comparison gradings between EURODIAB and WESDR systems

\begin{tabular}{|c|c|c|}
\hline Level & EURODIAB & WESDR final \\
\hline A. No retinopathy & Level 0 & Levels $10-15$ \\
\hline B. Mild non-proliferative & Level 1 & Levels $20-31$ \\
\hline $\begin{array}{l}\text { C. Moderate/Severe } \\
\text { non-proliferative }\end{array}$ & Levels 2-3 & Levels 41-55 \\
\hline $\begin{array}{l}\text { D. Proliferative } \\
\text { (or photocoagulated) }\end{array}$ & Levels 4-5 & Levels $60-80$ \\
\hline E. Cannot be graded & unassessable & Level $88+12$ \\
\hline
\end{tabular}

Table 4. Comparability and concordance of 2 -field grading by verified severity level

\begin{tabular}{llclcc}
\hline & & $\begin{array}{l}\text { Number } \\
\text { of eyes }\end{array}$ & $\begin{array}{l}\text { Number of cases of 2-field agree- } \\
\text { ment and concordance }\end{array}$ \\
\cline { 3 - 6 } & & & $\begin{array}{l}\text { no grader } \\
\text { agreed }\end{array}$ & $\begin{array}{l}\text { 1-2 graders } \\
\text { agreed }\end{array}$ & $\begin{array}{l}3-5 \text { graders } \\
\text { agreed }\end{array}$ \\
\hline Verified & $\mathrm{A}$ & 5 & 0 & 0 & 5 \\
level & $\mathrm{B}$ & 5 & 0 & 1 & 4 \\
& $\mathrm{C}$ & 20 & 1 & 2 & 17 \\
& $\mathrm{D}$ & 16 & 2 & 4 & 10 \\
& $\mathrm{E}$ & 2 & 0 & 1 & 1 \\
\hline \multirow{5}{*}{ Percent of total } & $(100 \%)$ & $(6 \%)$ & $(17 \%)$ & $(77 \%)$ \\
\hline
\end{tabular}

Results of comparability and concordance are shown in Table 4. Overall, of the 48 eyes compared, at least one grader (out of five) using the new system agreed with the verified level in $45(94 \%)$ cases and at least three agreed with the level in $37(77 \%)$ cases.

In the 41 eyes with diabetic retinopathy (verified levels B to D), when severity was expressed in three groups: mild background (level B), moderate/severe background (level C) and proliferative/photocoagulated (level D), at least one grader using the new system matched the verified results in 38 out of 41 $(93 \%)$ eyes and three or more graders matched in 31 $(76 \%)$ eyes.

Table 4 shows three cases where the verified severity level was not allocated by even one grader using the 2 -field $45^{\circ}$ grading. In two of the three cases early new vessels elsewhere, visible on the $30^{\circ}$ pictures, lay only outside the $45^{\circ}$ standard fields causing the assignment of inappropriate levels (i.e. non-proliferative). In the third case a patch of intraretinal microvascular abnormalities, graded on the $30^{\circ}$ photographs fell outside the two standard $45^{\circ}$ fields and caused the inappropriate allocation of level B (by all graders) in place of the verified level $C$.

Table 4 shows a further eight cases where only either one or two graders agreed with the verified level. Four of these involved level D (i.e. proliferative or photocoagulated) eyes. In three of these cases the eyes were correctly assigned as proliferative by two of the five graders but were incorrectly assigned as 
Table 5. Individual grader agreements by verified severity level

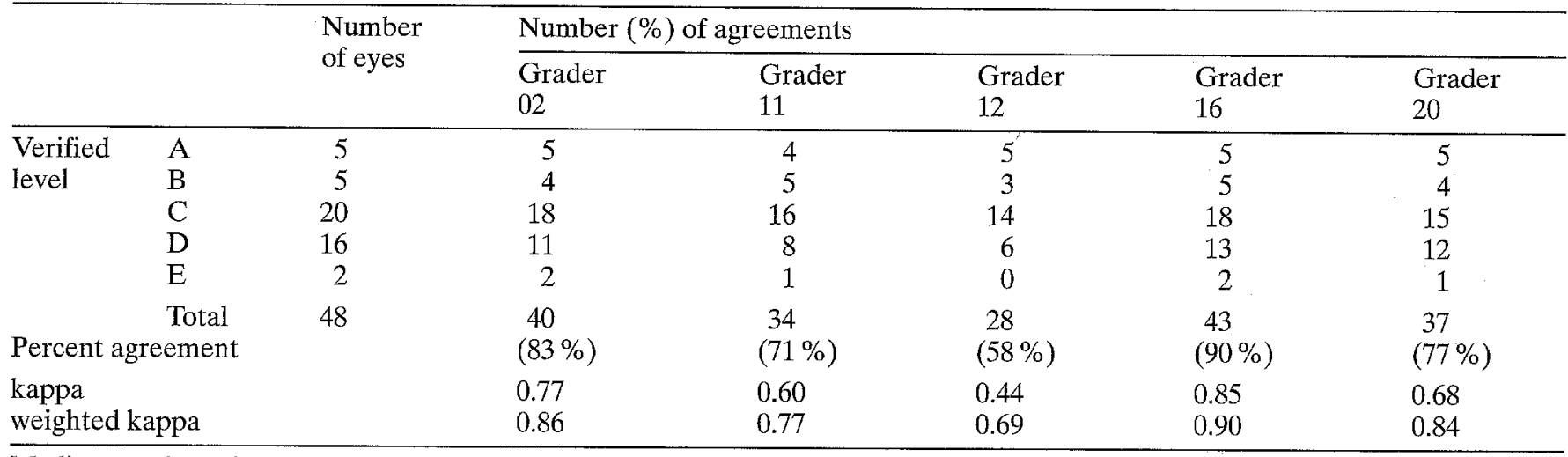

Median number of agreements $95 \%$ CI: 37.0; 27.4-45.4 Mean (SD) number of agreements $36.4 \pm 5.8$

Median unweighted kappa value $95 \%$ CI: $0.68 ; 0.42-0.92$ Mean (SD) unweighted kappa value $0.67 \pm 0.16$

non-proliferative by the remaining three. The proliferative lesions were therefore visible within the $45^{\circ}$ fields, but were misgraded by three graders.

In the remaining one of these four cases, small scars from previous photocoagulation, visible only on the edge of a $45^{\circ}$ field, were missed by four out of the five graders. The corresponding $30^{\circ}$ pictures showed quite extensive peripheral photocoagulation.

The lower image magnification of $45^{\circ}$ fields relative to $30^{\circ}$, caused lesions to be incorrectly graded by some (but not all) graders on some occasions. Similarly the absence of stereopsis, available in the $30^{\circ}$ system, caused occasional inaccuracies from some, but not all graders.

Details of the individual graders' exact agreements against the verified results are presented in Table 5 and are entirely consistent with their relative experience. The median number of agreements across graders was 37 of 48 (95\% CI: 27.4, 45.4), the median unweighted kappa value 0.68 (95\% CI: $0.42,0.92)$ and median weighted kappa value 0.84 (95\% CI: 0.68 , 0.94 ) when a weighting of 0.5 was assigned to those cases one-step displaced from the diagonal.

Observer variation validation. During the course of the assessment of clinical photographs, a stratified sample of 252 patients (approximately $10 \%$ of the total number) were randomly selected for regrading by the primary grader for measurement of within observer variation. A second, independent reader also carried out grading of a further 123 patients' photographs (approximately a $5 \%$ sample) for between observer assessment. Selection of both samples was based on a $50 \%: 40 \%: 10 \%$ mix of none, background and proliferative retinopathy, respectively.

Observer variation - results. Gradings were compared using three summary levels of retinopathy: none,
Median weighted kappa value $95 \%$ CI: $0.84 ; 0.68-0.94$ Mean (SD) weighted kappa value $0.81 \pm 0.08$

(weighting $=0.50$ for 1 -step displaced from true diagonal)

Table 6. Observer agreement

\begin{tabular}{lcccr}
\hline Between observer & $\begin{array}{c}\text { agreement } \\
\text { No reti- } \\
\text { nopathy }\end{array}$ & $\begin{array}{c}\text { Back- } \\
\text { ground }\end{array}$ & $\begin{array}{l}\text { Prolife- } \\
\text { rative }\end{array}$ & Total \\
\hline No Retinopathy & 57 & 4 & 0 & 61 \\
Background & 6 & 42 & 0 & 48 \\
Proliferative & 1 & 0 & 13 & 14 \\
Total & 64 & 46 & 13 & 123 \\
\hline kappa 0.85 (95\% CI 0.80-0.90) & & & \\
Within observer & agreement & & & \\
No retinopathy & 117 & 4 & 0 & 121 \\
Background & 18 & 82 & 0 & 100 \\
Proliferative & 0 & 4 & 27 & 31 \\
Total & 135 & 90 & 27 & 252 \\
\hline
\end{tabular}

kappa $0.83(95 \%$ CI $0.76-0.89)$

background and proliferative. Results from within $(n=252)$ and between $(n=123)$ observer agreement gradings are shown in Table 6.

Measures of agreement were assessed by the (unweighted) kappa coefficient $(\mathrm{K})$ [8], ranging from 0 for no agreement better than chance to 1 for perfect agreement, 'banded' in 0.2 (arbitrary unit) bands [9]. For within observer variation, a kappa coefficient of 0.85 (95\% CI: $0.80-0.90$ ) was achieved and for between observers 0.83 (95\% CI: 0.76-0.89), indicating 'very good' agreement in both assessments of variation.

\section{Discussion}

We present the methodology for two-field $45^{\circ}$ retinal photography and detail the development of a novel, accurate and repeatable grading system for the assessment of diabetic retinopathy from such photographs. 
The photographic protocol and grading system have been compared directly with the recognised 'gold standard' system of 7-field stereo retinal photography; assessed using techniques derived for the Diabetic Retinopathy Study and derivatives. The method described in this report compared favourably with this recognised system and proved to be acceptably repeatable and accurate.

On three occasions during the comparison of systems, lesions visible on the 7 -field $30^{\circ}$ photographs occurred only outside the 2-field $45^{\circ}$ photographs and hence were not visible to the graders using this system. As a result in two of these cases the eye was assigned as non-proliferative rather than proliferative and in the other case as mild rather than moderate/severe non-proliferative. Similarly however, on one occasion during the comparison assessments, new vessels were present only outside the 7 -field $30^{\circ}$ photographs (directly nasally to the optic disc) but were detected using the 2 -field protocol as described herein. Unfortunately such methodological errors will occur occasionally in any recording system such as this when compared to another possibly less 'restricted' system.

The lower image magnification of the $45^{\circ}$ system relative to the $30^{\circ}$ system and/or the lack of stereopsis on $45^{\circ}$ photography caused some grader inaccuracies, though on no occasion did all five graders concurrently miss or misclassify a lesion because of it.

Across the five graders, the verified level was agreed with on average in $76 \%$ of eyes. Differences between graders, as expected, did occur during the comparison and reflected the relative experience of the graders. The grader achieving the least number of exact agreements with the verified level (grader 12: $28 / 48$ agreements) was at the time still under training with less than 2 years of service. Similarly, the highest agreements (grader 16: $43 / 48$ and grader 02: 40/48) were senior graders, each with more than 10 years of experience.

The systems described are comparatively simple to apply and should prove useful additions to the currently accepted methodologies for the assessment of diabetic retinopathy. This is particularly the case in multicentre clinical trials when consistently high quality 7 -field $30^{\circ}$ stereo retinal photography on large numbers of patients would be difficult to achieve.

This 2-field photography and grading system is suitable for large epidemiological studies involving centres where retinal photography is not used regularly. It potentially could be useful in studies of noninsulin-dependent diabetes mellitus, as these patients are less likely to have proliferative retinopathy, with the caveat that as no stereopsis is available, maculopathy per se could not be fully assessed using this system.
Acknowledgements. We thank all the patients who took part in the study. The study was part of the EURODIAB Concerted Action Programme financially supported by the Commission of the European Communities. Additional financial support was received from ICI UK, Fidia, Bayer, Miles-Ames, Novo Nordisk and Pfizer.

Dr. J.Stephenson provided analyses on portions of the data.

\section{EURODIAB Investigators:}

B. Karamanos, C.Tountas, A.Kofinis, K. Petrou, N. Katsilambros, Hippokration Hospital, Athens, Greece.

R. Giorgino, M. Cignarelli, M. L. De Cicco, I. Ramunni, Istituto di Clinica Medica, Endocrinologia e Malattie Metaboliche, Universitá di Bari, Bari, Italy.

C.Ionescu-Tirgoviste, C.M. Iosif, D.Pitei, S. Buligescu, Clinic of Diabetes, Nutrition and Metabolic Diseases, Bucharest, Romania.

G. Tamas, Z. Kerenyi, A. M. Ahmed, J. Toth, P. Kempler, Tetenyi Teaching Hospital and Semmelweis University, Budapest, Hungary.

Se.Muntoni, M. Songini, M. Stabilini, M.Fossarello, S.Pintus, Sä. Muntoni, Ospedale San Michele, Cagliari, Italy.

J.B.Ferriss, C.C.Cronin, A.E. Whyte, P.E.Cleary, Cork Regional Hospital, Cork, Ireland.

M. Toeller, A.Klischan, T.Forst, F.A.Gries, Diabetes Forschungsinstitut, and W.Wagener, Medizinische Klinik E, Universität Düsseldorf, Germany.

R. Rottiers, H. Priem, University Hospital of Grent, Belgium.

P.Ebeling, M.Sinisalo, V.A. Koivisto, University Hospital of Helsinki, Finland.

B. Idzior-Walus, B. Solnica, L. Szopinska-Ciba, K. Solnica, University School of Medicine, Krakow, Poland.

H.M.J.Krans, H.H.P.J.Lemkes, J.J. Jansen, J. Brachter, University Hospital of Leiden, The Netherlands.

J. Nuñes-Correa, J. Boavida, Portuguese Diabetic Association, Lisbon, Portugal.

G. Michel, R. Wirion, Centre Hospitalier, Luxembourg.

A.J.M. Boulton, H. Ashe, D. J.S. Fernando, Manchester Royal Infirmary, UK.

G. Pozza, G. Slaviero, G. Comi, B. Fattor, F. Bandello, Ospedale San Raffaele, Milan, Italy.

H.Mehnert, A.Nuber, H.Janka, City Hospital Schwabing, Munich, Germany.

D.Ben Soussan, M.-C.Fallas, P.Fallas, Centre Hospitalier de Valenciennes, France.

E.Jepson, S. McHardy-Young, Central Middlesex Hospital, and J.H.Fuller, D. J. Betteridge, M. Milne, University College Hospital, London, UK.

G. Crepaldi, R. Nosadini, Instituto di Medicina Interna, Padua, Italy.

G. Cathelineau, B. Villatte Cathelineau, M. Jellal, N. Grodner, P. Gervais Feiss, Hospital Saint-Louis, Paris, France.

F. Santeusanio, G. Rosi, M. R. M. Ventura, C. Cagini, C. Marino, Instituto di Patologia Medica, Policlinico, Perugia, Italy.

R. Navalesi, G.Penno, R. Miccoli, M.Nannipieri, S.Manfredi, Instituto di Clinica Medica II, Pisa, Italy.

G. Ghirlanda, P.Cotroneo, A. Manto, C. Teodonio, A. Minella, Universitá Cattolica del Sacro Cuore, Rome, Italy.

J.D. Ward, S. Tesfaye, C.Mody, C. Rudd, Royal Hallamshire Hospital, Sheffield, UK.

G. M.Molinatti, F.Vitelli, M.Porta, G.F.Pagano, P.Cavallo Perin, P. Estivi, R. Sivieri, Q. Carta, G. Petraroli, Clinica Medica B, Patologia Medica, Ospedale Molinette, and Ospedale "Agnelli", Turin, Italy. 
N.Papazoglou, G.Manes, G. Triantaphyllou, A. Ioannides, G.Skazagar, I.Kontogiannis, General Hospital of Thessaloniki, Greece.

M.Muggeo, V.Cacciatori, F.Bellavere, P.Galante, M.L. Gemma, Cattedra di Malattie del Metabolismo, Verona, Italy.

K. Irsigler, H. Abrahamian, C. Gurdet, B. Hornlein, C.Willinger, Hospital Vienna Lainz, Austria.

S. Walford, E. V. Wardle, S. Hughes, New Cross Hospital, Wolverhampton, UK.

G. Roglic, Z.Resman, Z.Metelko, Z.Skrabalo, Vuk Vrhovac Institute for Diabetes, Zagreb, Croatia.

Steering committee members: J.H.Fuller (London), H. Keen, Chairman (London), H.M.J.Krans (Leiden), R. Navelesi (Pisa), A.-K.Sjølie (Århus), J.M.Stephenson (London), M.Toeller (Düsseldorf), G.-C.Viberti (London), J. Ward (Sheffield).

Coordinating Centre: J.H.Fuller, J.Stephenson, T.Partridge, M. Milne, UCL Medical School, UK.

Central Laboratories: G.John, The Royal London Hospital, and G.-C.Viberti, M.Mattock, A.Collins, A.Dredge, R. Sharp, Guy's Hospital, London, UK.

Retinopathy Coordination: A.-K.Sjølie, Århus University Hospital, Denmark.

Retinopathy Grading Centre: E.Kohner, S.Aldington, S. Cockley, Hammersmith Hospital, London, UK.

\section{References}

1. The EURODIAB IDDM Complications Study Group (1994) Microvascular and acute complications in insulin dependent diabetes mellitus: the EURODIAB IDDM Complications Study. Diabetologia 37: 278-285

2. Diabetic Retinopathy Research group (1981) Report 7. A modification of the Airlie House classification of diabetic retinopathy. Invest Ophthalmol Vis Sci 21/2: 244-251

3. The Wisconsin Epidemiologic Study of Diabetic Retinopathy (1984) III. Prevalence and risk of diabetic retinopathy when age at diagnosis is 30 or more years. Arch Ophthalmol 102: 527-532

4. Early Treatment Diabetic Retinopathy Study (1985) Report number 1. Photocoagulation for diabetic macular edema. Arch Ophthalmol 103: 1796-1806

5. The Diabetes Control and Complications Trial (DCCT) (1986) Design and methodological considerations for the feasibility phase. Diabetes 35: 530-545

6. Klein BEK, Davis MD, Segal P et al (1984) Diabetic retinopathy. Assessment of severity and progression. Ophthalmology 91: 10-17

7. Early Treatment Diabetic Retinopathy Study (ETDRS) manual of operations (1979) ETDRS coordinating center, University of Maryland, Department of Epidemiology and Preventative Medicine, Division of Clinical Investigation, 600 Wyndhurst Ave, Baltimore, Maryland 21210

8. Altman DG (1991) Some common problems in medical research. Inter-rater agreement. Practical statistics for medical research. Chapman, London, pp 403-409

9. Landis JR, Koch GC (1977) The measurement of observer agreement for categorical data. Biometrics 33: 159-174 